\title{
Membranes with a particulate morphology prepared by a dry-wet casting process
}

\author{
Tai-Horng Young ${ }^{\mathrm{a}, *}$, Dong-Tsamn Lin ${ }^{\mathrm{b}}$, Li-Yen Chen $^{\mathrm{c}}$, Yao-Huei Huang ${ }^{\mathrm{c}}$, Wen-Yen Chiu ${ }^{\mathrm{c}}$ \\ anstitute of Biomedical Engineering, College of Medicine and College of Engineering, National Taiwan University, Taipei 10016, Taiwan \\ ${ }^{\mathrm{b}}$ Department of Laboratory Medicine, College of Medicine, National Taiwan University, Taipei 10016, Taiwan \\ ${ }^{\mathrm{c}}$ Department of Chemical Engineering, National Taiwan University, Taipei 10764, Taiwan
}

Received 22 April 1998; received in revised form 21 August 1998; accepted 7 October 1998

\begin{abstract}
The effect of the evaporation step on the occurrence of particles in poly(ethylene-co-vinyl alcohol) (EVAL) membranes cast from DMSO solutions via the dry/wet process were studied. The structure of the EVAL membranes can be changed from an asymmetrical structure consisting of a dense skin layer and finger-like macrovoids in the sublayer to a skinless and symmetric structure by constituent particles bonded to each other. From the analysis of the membrane formation mechanism, the competition of different phase separation process during membrane formation is most important. The onset of the phase separation may be either liquid-liquid demixing or solid-liquid demixing, which determines the resulting membrane properties. Directly immersing the casting solution into a water bath, liquid-liquid demixing is of considerable importance during the phase separation of the solutions. As a result of the lower activation energy for nucleation, liquid-liquid demixing can precede solid-liquid demixing even in cases where solid-liquid demixing is favored thermodynamically. By using the evaporation process, the phase separation proceeds slowly via solid-liquid demixing and thus leads to a particulate morphology in the membrane. This suggests that the evaporation step cause crystallization of EVAL molecules from the casting solution to inhibit the macrovoid formation. In addition, the duration of the evaporation step is shown to have a strong influence on the disappearance of particles. The results presented here offer a qualitative basis for the development of membranes with a particulate morphology. (C) 1999 Elsevier Science Ltd. All rights reserved.
\end{abstract}

Keywords: Dry/wet process; Particulate morphology; Membrane formation

\section{Introduction}

Phase inversion process has been a standard technique to prepare polymeric membranes [1]. The mechanism of this process is the induction of phase separation in an initially homogeneous polymer solution through a change in the composition of the solution. With crystallizable polymers, two types of phase separation may occur during the membrane formation by phase inversion precipitation [24]. Liquid-liquid demixing results in the typical cellular morphology with pores from polymer-poor phase surrounded by the membrane matrix from polymer-rich phase. Solid-liquid demixing is from crystallizable segments of the polymer to form membranes by linking of particles. In our previous studies, particulate membranes prepared from poly(ethylene-co-vinyl alcohol) (EVAL)

\footnotetext{
* Corresponding author. Tel.: + 886223970800 , ext. 1455; fax: + 886 223940049.

E-mail address: thyoung@ha.mc.ntu.edu.tw (T.-H. Young)
}

were investigated for possible use in plasma protein separation and microfiltration [5,6]. It was found that particulate EVAL membranes exhibited not only high permeation rates with respect to albumin and immunoglobulins but also good selectivity between these components. Thus, this work was performed to elucidate the formation mechanism of particulate membranes that have the potential to be used in the plasma filtration.

In order to develop the membrane formation mechanism, an equilibrium phase diagram of polymer/solvent/nonsolvent is necessary. The equilibrium thermodynamics of the ternary system water-dimethyl sulfoxide(DMSO)-EVAL has been described previously [7]. As shown in Fig. 1, there are two regions of metastability: one with respect to solid-liquid demixing and the other with respect to liquidliquid demixing. This indicates that phase separation may occur both by the processes of liquid-liquid demixing and crystallization to yield membranes exhibiting characters from both types of phase separations. Cheng pointed out that the sequence of precipitation events affects most 


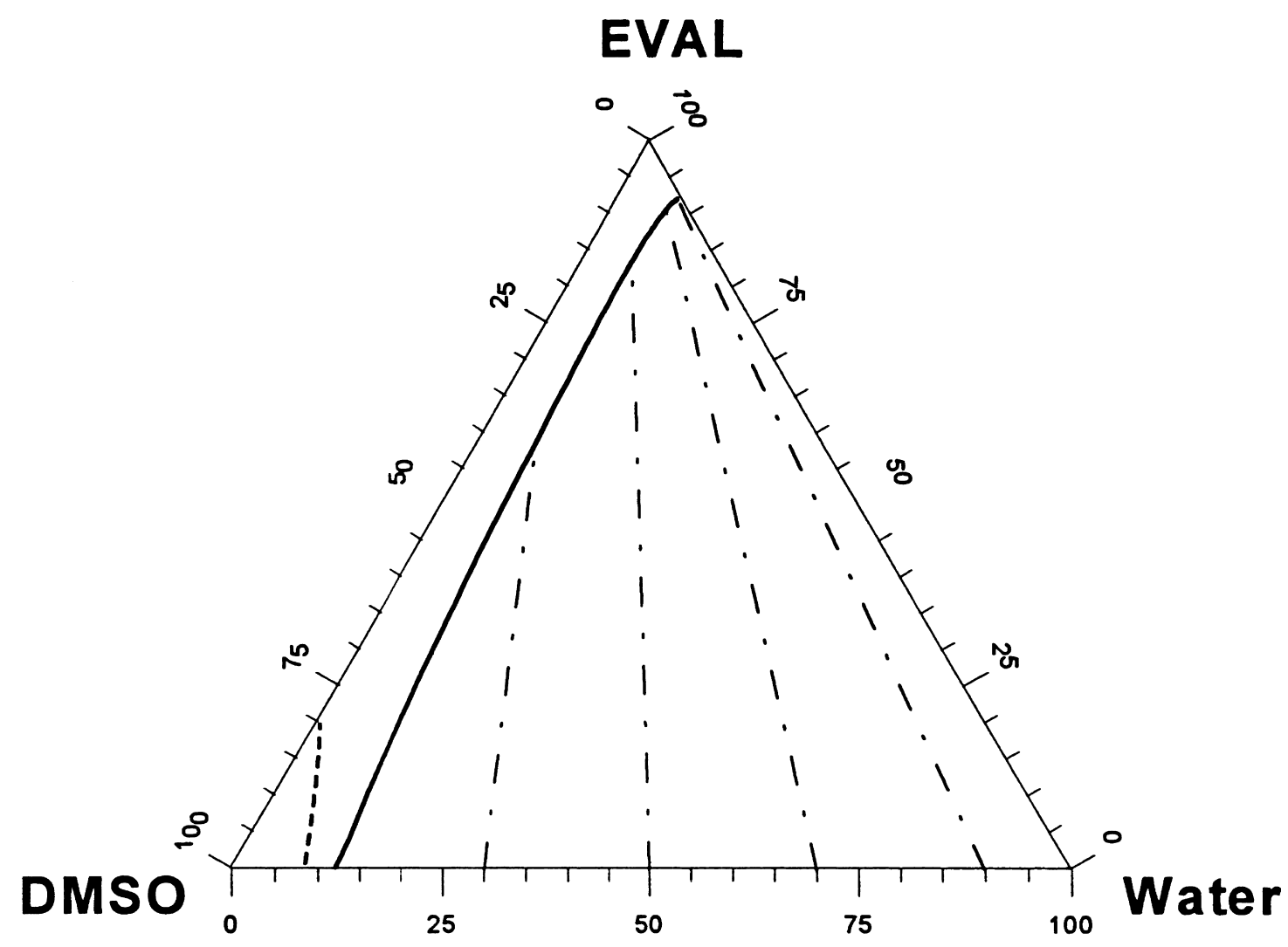

Fig. 1. Phase diagram of water-DMSO-EVAL at $25^{\circ} \mathrm{C}$. (- - - -: crystallization equilibrium line; —: binodal boundary; - - —: tie line).

significantly the relative weight of both types of phase separation on the formed membrane morphology [3]. In general, predictions of the sequence of precipitation events are by considerations of the thermodynamic (phase behavior) and kinetic (mass transfer) aspects of the phase inversion process. In the phase diagram of water-DMSO-EVAL, the binodal boundary is located inside the crystallization equilibrium line, which means that solid-liquid demixing is thermodynamically more favorable. However, crystallization is a slower process than liquid-liquid demixing because it requires reorientation of the polymer chain into the crystalline lattice. Therefore, the kinetic factor has a negative effect on the crystallization under normal conditions. Consequently, the slow process of crystallization can be easily surpassed when the composition changes in the membrane formation are rapid. As a result, the liquid-liquid demixing dominates the phase separation process during the membrane formation. This is why the crystallization phenomenon has often been overlooked in most membrane literature. In fact, very few reports exist in the open literature describing the formation mechanism of membrane morphology in terms of crystallization during the precipitation process $[2-4,7]$.

This article reports a study of the preparation of EVAL membranes by the dry/wet phase inversion technique. The phase separation can be induced by the evaporation of solvent from the casting solution in the dry step. Therefore, the polymer in the membrane solution has a longer period to become supersaturated with respect to crystallization and crystallization nucleation is probably to occur in the dry step. Although there are several models proposed to describe the solvent evaporation pertinent to the dry step [8-10], their principal research efforts are dedicated to amorphous polymers. These theories are not satisfactory in explaining the formation of membranes from crystallizable polymers. Until now only few studies have been devoted to crystallization in relation to membrane formation in a dry/wet process [11].

In this work, the membrane structure prepared by a dry/ wet casting process consisted of constituent particles bonded to each other, similar to the particulate EVAL membranes prepared by directly immersing the EVAL solution into a 1-octanol bath [6]. However, a potential problem associated with the use of 1-octanol as nonsolvent is their low solubility in water that exacerbates its subsequent extraction. Indeed, the removal of 1-octanol from the membrane required extensively washing by a series of washing steps. Therefore, to employ the dry/wet process to prepare membranes is a possible means of circumventing problems associated with the use of 1-octanol nonsolvent. 


\section{Experiments}

\subsection{Membrane preparation}

The membrane material studied in this work is EVAL copolymer which contains ca. $56 \mathrm{~mol} \%$ vinyl alcohol (intrinsic viscosity $=0.87 \mathrm{dl} / \mathrm{g}$, measured $M_{\eta}=56,000 \mathrm{~g} /$ mol) [7]. This polymer was kindly supplied by Kuraray Co. Ltd., Japan. DMSO of extra pure reagent grade (Nacalai Tesque, Kyoto, Japan) was used as-received. Double distilled and de-ionized water was used as the precipitation bath at room temperature $\left(23 \pm 2^{\circ} \mathrm{C}\right)$.

An appropriate amount of EVAL was dissolved in DMSO to form a $25 \%(\mathrm{w} / \mathrm{w})(23 \%(\mathrm{v} / \mathrm{v}))$ polymer solution. Although the solution is thermodynamically unstable which is located in the crystallization region in the phase diagram (Fig. 1), the solution can maintain its state for at least two weeks if no humidity is present. This solution was dispersed uniformly on a glass plate (ca. $100 \mathrm{~m}$ ) by an autocoater (KCC303, RK Print-Coat Instruments, UK), and then phase-separated to form a membrane. For comparison, all membranes were prepared from the same polymer solution and coagulant (water). The first membrane (termed $A$, hereinafter) was prepared by directly immersing the casting solution into a water bath without an evaporation step. The second membrane (termed $B$, hereinafter) and the third membrane (termed $C$, hereinafter) were subjected to a two-stage process of precipitation. After casting, the casting solution was immediately placed in a vacuum oven. Solvent evaporation was then evacuated at $6 \mathrm{cmHg}$ and room temperature for a predetermined time to reduce the DMSO content, after which the evaporated solution was immersed into a water precipitation bath where it remained until the phase inversion process was completed. The membrane was then washed with water to remove residual DMSO. The duration of evaporation is $5 \mathrm{~min}$ and one day for membranes $B$ and $C$, respectively.

\subsection{Membrane characterization}

The morphology of the membranes was examined on freeze-dried samples using a scanning electron microscope (SEM). Pure water flux and plasma ultrafiltration were determined using a $25 \mathrm{~mm}$ diameter Amicon Stirred Ultrafiltration Cell (Model 8010) connected to a compressed nitrogen gas source. The transmembrane pressure was equal to $0.5 \mathrm{kgf} / \mathrm{cm}^{2}$. The plasma used in this work was extracted from human blood of healthy donors. All filtration experiments were carried out at room temperature $(23 \pm$ $2^{\circ} \mathrm{C}$ ). After the permeate flux reaches a stable constant value (ca. $50 \mathrm{~min}$. after operation), the filtrate samples were collected for subsequent analysis. The albumin and the $\mathrm{IgG}$ content were measured by using the Array Protein System (Beckman) and the Nephlometer-Analyzer (Behring), respectively. In addition, a $100 \mathrm{ppm}$ of blue dextran (average molecular weight $=2000 \mathrm{kDa}$, Sigma) solution was filtered to check whether the membrane was defected. All tested membranes were found to reject the passage of this blue dextran.

\subsection{Light transmission experiment}

Light transmission experiments were performed to measure the time of the onset of phase separation of the casting solution in the precipitation bath. The principle of light transmission experiments is that the light transmittance of the casting solution would decrease with the appearance of optical inhomogenities, which can be induced by liquidliquid demixing or solid-liquid demixing. Therefore, the time that the light transmittance begins to drop can be used to represent the time of the onset of phase separation. To carry out the light transmission experiment, a lamp was placed above the coagulation bath as light source and a light detector beneath the coagulation bath was used to measure the light transmittance. For detailed experimental setup and procedures, one can refer to the work of Reuvers et al. [12]. In addition, the light transmittance for the casting solutions $B$ and $C$ prior to and after the evaporation process was compared to investigate the effect of the evaporation process on the membrane formation mechanism.

\section{Results and discussion}

\subsection{Membrane morphology}

Fig. 2 shows the structure of membrane $A$, which was prepared by directly immersing a casting solution into a water bath. An asymmetrical structure consisting of a dense skin layer and finger-like macrovoids in the sublayer was observed. Between macrovoids were revealed cellular morphologies characteristic of amorphous membranes. It is believed that this structure is a consequence of liquid-liquid demixing process. From Fig. 1, however, it can be found that mass exchange of solvent and nonsolvent brings the casting solution into a metastable state initially with respect to solid-liquid demixing, and then with respect to liquidliquid demixing. This suggests that liquid-liquid demixing can precede solid-liquid demixing even if solid-liquid demixing is favored thermodynamically [13]. This can be ascribed to the high activation energy needed for crystallization. Therefore, the induction time is not necessarily long enough to proceed solid-liquid demixing when the composition path crossed the crystallization equilibrium line. Consequently, solid-liquid demixing process does not take place prior to liquid-liquid demixing and the typical cellular morphology can be considered as a result of instantaneous liquid-liquid demixing.

Fig. 3 shows the structure of membrane $B$ that was prepared by a dry/wet phase inversion, including a 5-min evaporation step. The feature change of the membrane structure from a typical asymmetric morphology to a morphology with particles knitted together to maintain its 

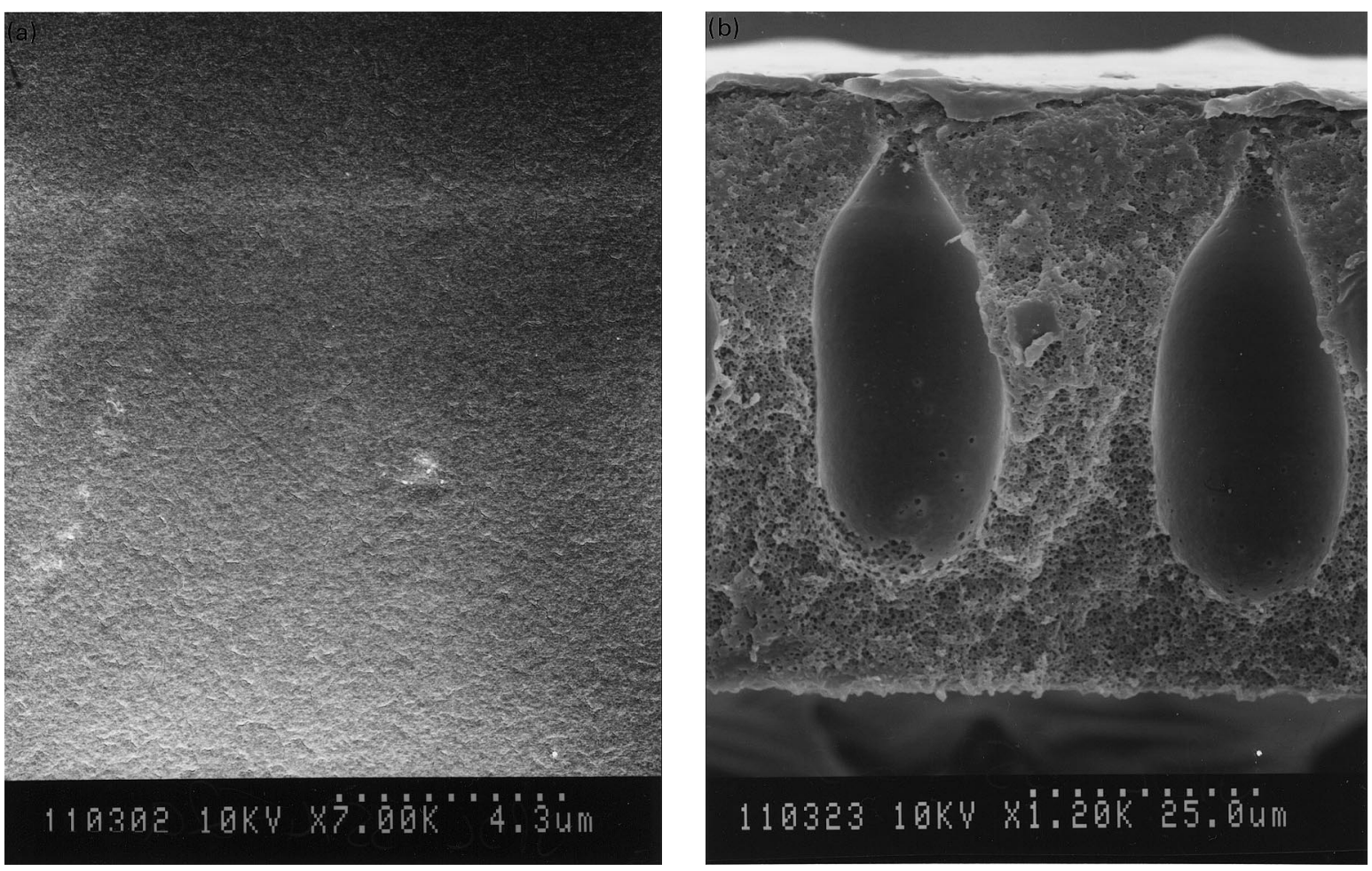

Fig. 2. SEM photomicrographs of membrane A: (a) top surface, (b) cross section.

mechanical strength was observed. The membrane is skinless and the pores between particles appeared to be open and connected. This indicated that the casting solution was phase-separated not because of the liquid-liquid demixing process. Particles dominating the membrane structure has also been observed for other polymers [14]. We agree with Reuvers et al. who thought that microcrystallites were responsible for the particle structure [14]. The detailed membrane formation mechanism is shown later.

In contrast, the membrane $C$ immersed into a water bath after the one-day evaporation showed a fairly dense structure without any particles existing in the cross section (Fig. 4). However, it can be noticed from a close look at the top layer of the membrane that the membrane has a special structure with superficial pores. By comparing Figs. 3 and 4 it is shown that particles will coalesce when the evaporation time is increased. This implies that the particles are somehow driven together to disappear in the boundary during the membrane formation.

\subsection{Light transmission}

The results of light transmission experiments for the various precipitation conditions are shown in Fig. 5. Clearly, instantaneous demixing takes place in all membrane formation processes. However, the light intensity of membrane $C$ decreases by only $50 \%$ after immersion for $30 \mathrm{~s}$. This may be because the dense structure resulting from the coalescence of particles is more transparent. In addition, there is almost no difference in light transmission for casting solutions $B$ and $C$ prior to and after the evaporation step. After evaporation, an originally transparent casting solution did not show a cloudy phenomenon.

\subsection{Water flux and plasma ultrafiltration}

Table 1 presents the results of determining the volumetric water flux and filtrate concentrations for plasma ultrafiltration at the transmembrane pressure of $0.5 \mathrm{kgf} / \mathrm{cm}^{2}$. As expected, the water flux actually reflects the morphology of the membrane. Membrane $A$ has larger finger-like macrovoids, but its skin layer is a region with very small pores that provide an effective barrier and thus has a lower water flux. Membrane $B$ is skinless and has a particulate morphology in which all voids between particles are interconnected, modifying membrane function. Hence, water molecules can flow through the continuous void channels with a rather low resistance. Conversely, upon increasing the evaporation period, no water flux for membrane $C$ is obtained under ultrafiltration conditions. The very low water permeability can be explained by the dense structure of membrane $C$ resulting from the coalescence of particles.

In order to examine the permselectivity of different membrane structures, plasma filtration experiments were carried out using human plasma as the feed in a dead-end filtration. Feed and filtrate samples were analyzed to yield the data of total protein, albumin and IgG concentrations. From Table 1, it can be seen that all proteins could not penetrate membranes $A$ and $C$, but albumin could pass through membrane $B$ to a significant degree. Further, IgG 


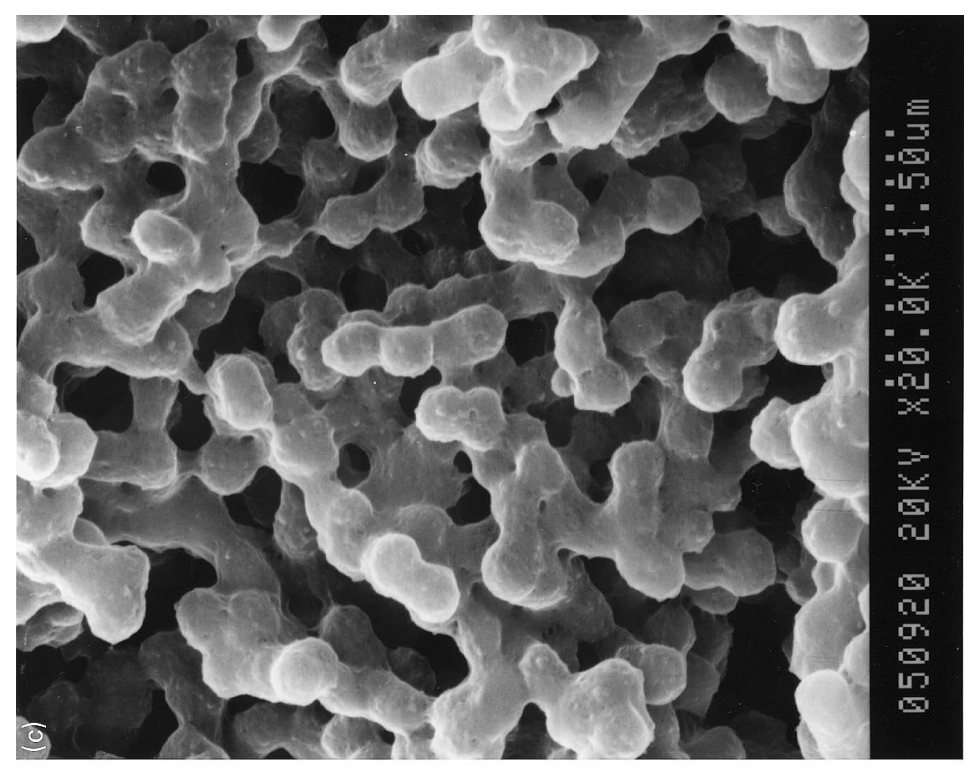

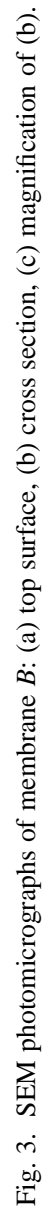

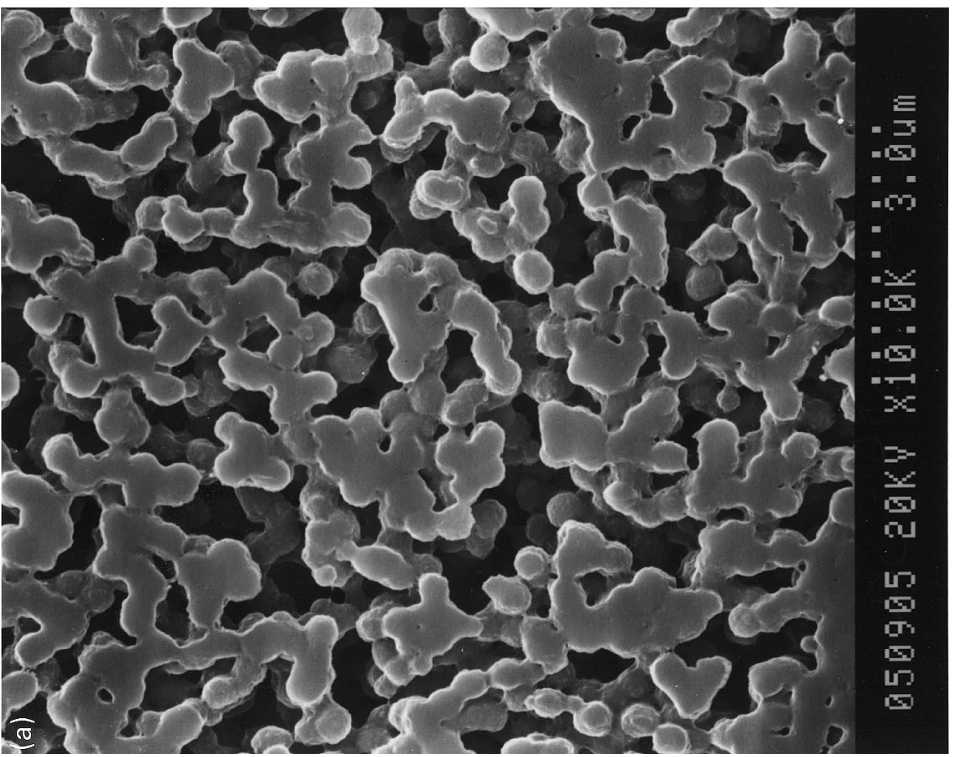




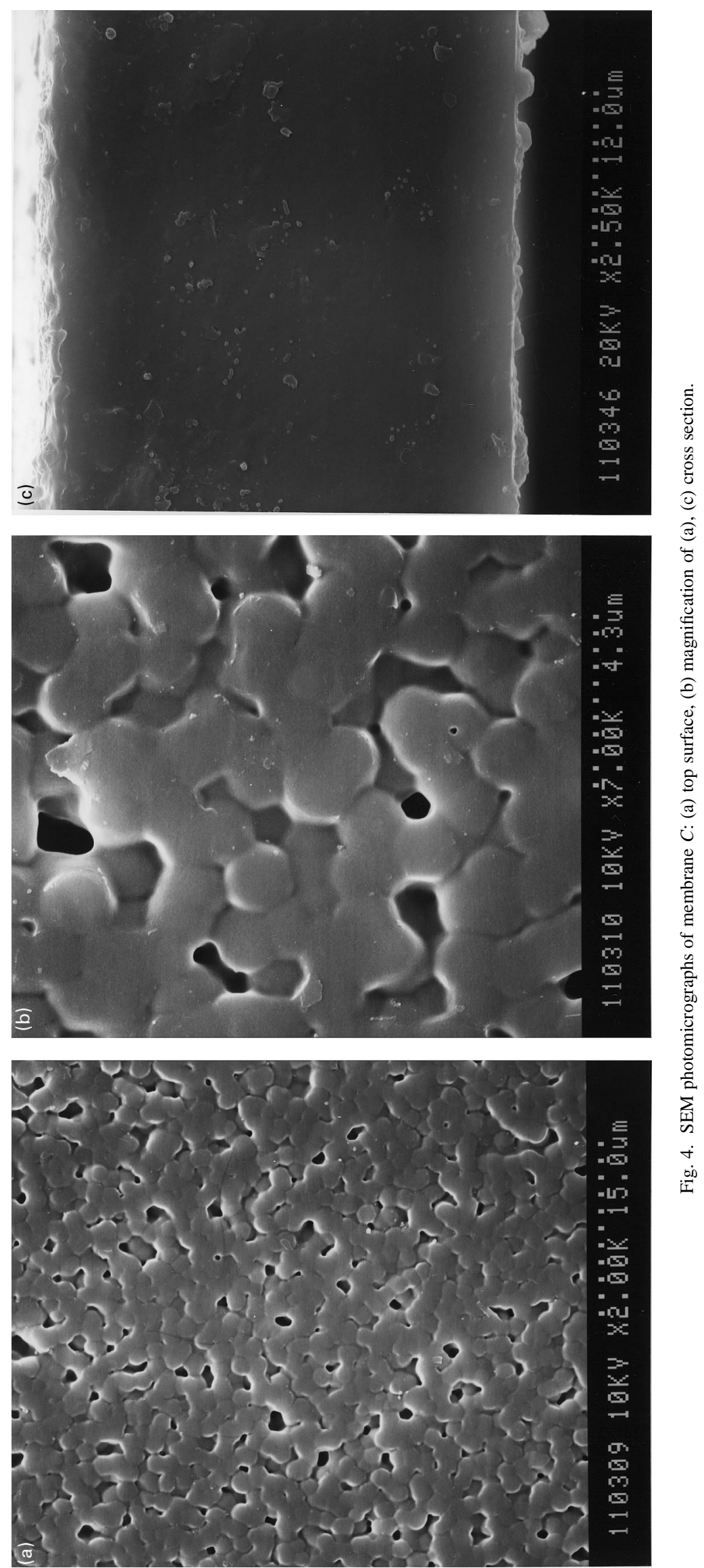




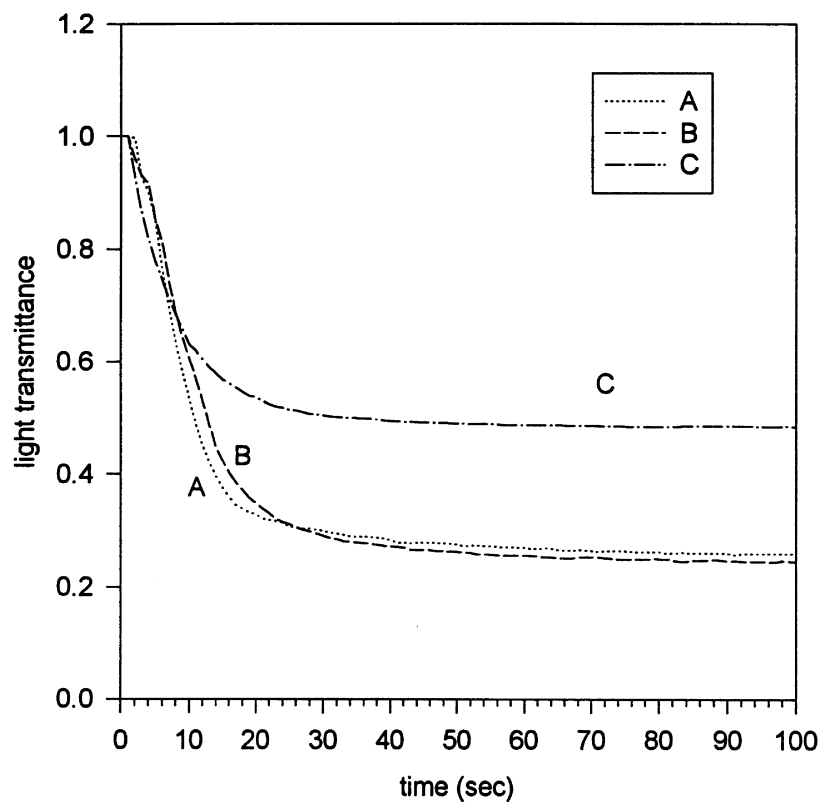

Fig. 5. The results of light transmission experiments for the various membranes.

is undetectable in the permeate for membrane $B$. As membrane $B$ is permeable to albumin while rejecting IgG, the pores in these particulate membranes can be considered between the dimension of albumin $(67 \mathrm{kDa})$ and $\mathrm{IgG}$ $(150 \mathrm{kDa})$. This suggests that the particulate membranes have the potential to be used in separating albumin from immunoglobulins.

\subsection{Membrane formation mechanism}

Both crystallization and liquid-liquid demixing are anticipated in a precipitation process for preparing EVAL membranes. This work shows that the membranes prepared without an evaporation process have finger-like macrovoids, while membranes prepared with evaporation of the cast solution in vacuum provide a symmetric particulate structure and particles with a narrow-size distribution. This morphological difference arises from the fact that their courses of precipitation are different. As initiation of liquid-liquid demixing is more rapid than nucleation of

Table 1

Water flux and filtrate concentrations for plasma ultrafiltration at a transmembrane pressure of $0.5 \mathrm{kgf} / \mathrm{cm}^{2}\left(\right.$ Feed: $\mathrm{TP}^{\mathrm{a}}=6.4 \mathrm{~g} / \mathrm{dl} ; \mathrm{Alb}^{\mathrm{b}}=3.8 \mathrm{~g} / \mathrm{dl}$; $\mathrm{IgG}=1340 \mathrm{mg} / \mathrm{dl}$ )

\begin{tabular}{lllll}
\hline Membrane & $\begin{array}{l}\text { Water flux } \\
(\mathrm{m} / \mathrm{s})\end{array}$ & \multicolumn{4}{l}{ Filtrate analysis } \\
\cline { 3 - 5 } & & $\mathrm{TP}(\mathrm{g} / \mathrm{dl})$ & $\mathrm{Alb}(\mathrm{g} / \mathrm{dl})$ & $\mathrm{IgG}(\mathrm{mg} / \mathrm{dl})$ \\
\hline $\mathrm{A}$ & $7.80 \times 10^{-7}$ & $\mathrm{ND}^{\mathrm{c}}$ & $\mathrm{ND}$ & $\mathrm{ND}$ \\
$\mathrm{B}$ & $4.65 \times 10^{-5}$ & 2.9 & 1.9 & $\mathrm{ND}$ \\
$\mathrm{C}$ & $\mathrm{ND}$ & $\mathrm{ND}$ & $\mathrm{ND}$ & $\mathrm{ND}$ \\
\hline
\end{tabular}

\footnotetext{
${ }^{\mathrm{a}} \mathrm{TP}=$ total protein.

${ }^{\mathrm{b}} \mathrm{Alb}=$ albumin.

${ }^{\mathrm{c}} \mathrm{ND}=$ not detectable.
}

polymer crystallization that requires a rearrangement of polymer, liquid-liquid demixing is kinetically favored and often initiates the precipitation process in the direct immersion process. Therefore, the thermodynamically possible phase transition (crystallization) is not significant in a real process. Conversely, the liquid micro-droplets forms is surrounded by polymer-rich phase and grow into the cellular morphology in the final membrane. Further, the polymerrich phase is always supersaturated with respect to crystallization; crystallization necessarily occurs within the twophase structure at the final stage. As crystallization occurs at a late stage at which liquid pores have fully developed, the morphology of the membrane is mostly determined by the liquid-liquid demixing process. Nonetheless, membranes prepared by an evaporation process result in a change in the phase separation process, yielding a particulate structure with no skin layer.

Considering the phase diagram and experimental results, membrane formation mechanism of the dry/wet process can be established. As a result of the low volatility of DMSO, the composition change in the evaporation process is rather slow and the evaporation of DMSO from the surface of the casting solution can be assumed to be compensated by DMSO diffusion from the casting solution interior to the surface. Therefore, the liquid-liquid demixing does not occur before the casting solution is immersed into the coagulation bath and changes in the composition over the whole cross section of the casting solution during evaporation are negligible. Consequently, when the driving force for crystallization increases with increasing EVAL concentration, the initiation of particle nuclei occurs along the entire casting solution virtually simultaneously. As the particle size requires the determination of the induction time before any developing nuclei appear, particles having a uniform size is evidence of simultaneous nucleation. In addition, low volatility of DMSO can reduce the possibility of a rapid composition change at the top layer of the membrane to form a dense skin layer. In this case, it is easy to correlate with membrane formation mechanism in the evaporation process with thermodynamic factors alone without considering kinetic factors.

Although the nuclei of particles are initiated in the stage of evaporation, these nuclei grow very slowly because the motion of EVAL molecules is restricted by high viscosity at low temperature and at high polymer concentration. In contrast, if the growth of crystallization is very rapid during the evaporation process, the light transmission intensity should decrease before immersing into a water bath. However, there is no difference of light transmission for casting solution prior to and after the evaporation process. This poses the particle nuclei do not grow very much in the evaporation process and at least the particle diameter is small compared with the wave length of the light.

A comparison of curves $a$ and $b$ in Fig. 5 shows that when the casting solution undergoes a solvent evaporation step, the demixing rate in the subsequent gelation step is still 
instantaneous. It is, however, not certain at this point whether the observed precipitation for the casting solution $B$ results from liquid-liquid demixing or from crystallization. If the growth of the particle is completed owing to crystallization, the precipitation may not be so fast. This suggests that liquid-liquid demixing may take place in the stage of particle growth. Thus, the growth of particles is assumed when EVAL molecules in the polymer-rich phase from the liquid-liquid demixing process move with a preferred chain orientation relative to a center (nucleus). Although the exact nature of particles has not yet been satisfactorily resolved, the particle can be considered as a semicrystalline state that is an amorphous matrix in which the nucleus is embedded. Therefore, liquid-liquid demixing only plays an important role in the wet stage which crystallization nuclei had initiated in the dry stage. Accordingly, after the immersion of the evaporated casting solution in a nonsolvent, the generated particle nuclei distribute in the casting solution and restrict the effect of liquid-liquid demixing on the membrane morphology. This implies the formation of nuclei in the dry process has determined the resulting membrane characteristics. In addition, these particles were not termed spherulites because no Maltese cross was observed under cross-polarized. This is an indirect evidence for the existence of the polymer-rich phase that had formed at the stage of particle growth.

Concerning the influence of evaporation time on the membrane structure, the evaporation time in the dry process affects the morphology of the resulting membranes directly. After the casting solution has been immersed in a water bath, these nuclei grow radially until their fronts meet and join with adjacent particles. From Fig. 4, membrane $C$ shows a particulate surface layer supported by a dense sublayer. Hence, it is suggested that the coalescence process between neighboring particles leads to a dense structure. As the solvent leaves the casting solution, the particle centers approach each other. When two particles are in contact, the amorphous morphology supplies the coalescence of particles by interdiffusion of polymer chain segments through a reptation mechanism and the surface tension supplies the necessary shearing stress [15]. In this case, the polymer molecules are relatively soft in a nascent membrane by the presence of residual solvent to produce sufficient mobility to reorganize the membrane structure before the solvent has left completely, so the coalescence process is promoted. Therefore, the longer the evaporation time the more the nuclei formed in the evaporated casting solution and the more the coalescence of particles. However, the too long evaporation time may be deleterious to the separation properties of the particulate membrane, rendering the membrane structure less porous.

\section{Conclusions}

This study of the dry/wet process for the formation of membranes cast from EVAL/DMSO solutions into a water precipitation bath clearly indicates that the evaporation step strongly influences the final membrane structure. It is suggested that such cast membranes can undergo phase separation by crystallization-induced (solid-liquid) demixing process. Therefore, the liquid-liquid demixing process can be suppressed and a particulate membrane was prepared. From the permeation measurements, the particulate membrane exhibits a better permeation ability and a proper permselectivity between albumin and IgG. This provokes us to continue our research for a better membrane capable of separating albumin from the globulins. Further, the final membrane structure is sensitive to the evaporation time with significant membrane property changes expected as a result of a one-day evaporation period. The relationship between the duration of the evaporation step and the coalescence of particles, can thus be exploited to govern the transport characteristics of the final membranes. However, it is important to keep in mind that the conclusions summarized earlier are specific to the dry/wet casting of the crystallizable EVAL polymers. Other studies suggest that the amorphous membrane structure is quite different in the dry/ wet cast process [16]. Clearly, particulate membranes reported here are believed to be observed in other membrane formation systems for which two type of transitions could be observed in the phase diagram.

\section{Acknowledgements}

The authors thank the National Science Council of the Republic of China for their financial support of project NSC 86-2216-E-002-003.

\section{References}

[1] Kesting RE. Synthetic polymeric membranes. New York: Wiley, 1985.

[2] Bulte AMW, Folkers B, Mulder MHV, Smolders CA. J Appl Polym Sci 1993;50:13.

[3] Cheng LP, Dwan AW, Gryte CC. J Polym Sci Polym Phys 1995;33:211.

[4] Bulte AMW, Mulder MHV, Smolders CA, Strathmann H. J Membrane Sci 1996;121:37.

[5] Lin DT, Cheng LP, Kang YJ, Chen LW, Young TH. J Membrane Sci 1998;140:185.

[6] Cheng LP, Lin HI, Chen LW, Young TH. Polymer 1998;39:2135.

[7] Young TH, Lai JY, You WM, Cheng LP. J Membrane Sci 1997;128:55.

[8] Castellari C, Ottani S. J Membrane Sci 1981;9:29.

[9] Zeman L, Fraser T. J Membrane Sci 1994;87:267.

[10] Huang RYM, Feng X. J Appl Polym Sci 1995;57:613.

[11] Wijmans JG, Rutten HJJ, Smolders CA. J Polym Sci Polym Phys 1985;23:1941

[12] Reuvers AJ, van der Berg JWA, Smolders CA. J Membrane Sci 1987;34:45.

[13] van de Witte P, Boorsma A, Esselbrugge H, Dijkstra PJ, van de Berg JWA, Feijen J. Macromolecules 1996;29:212

[14] Reuvers AJ, Altena FW, Smolders CA. J Polym Sci Polym Phys 1986;24:793.

[15] Pinnau I, Koros WJ. J Polym Sci Polym Phys 1993;31:419.

[16] Paulsen FG, Shojaie SS, Krantz WB. J Membrane Sci 1994;91:265. 